\title{
A Flow-Diverting Stent Is Not a Pressure-Diverting Stent
}

\author{
J.J. Schneiders, E. VanBavel, C.B. Majoie, S.P. Ferns, and R. van den Berg
}

\begin{abstract}
SUMMARY: The approach for treatment of large and fusiform intracranial aneurysms has evolved from stent-assisted coiling to treatment with flow-diverting stents. The treatment results for these stents are promising; however, early postprocedural aneurysm rupture has been described. The exact cause of rupture is unknown but might be related to intra-aneurysmal flow and pressure changes. We measured intra-aneurysmal pressure before, during, and after placement of a flow-diverting stent by using a dual-sensor guidewire. The pressure inside the aneurysm momentarily decreased during placement but was restored to baseline values within minutes. The flow-diverting stent does not seem to protect the aneurysm from the stress induced by pressure or pressure changes within the lumen.
\end{abstract}

ABBREVIATIONS: ICA = internal carotid artery; $\mathrm{MCA}=$ middle cerebral artery

$\mathbf{T}$ he approach for treatment of large and fusiform intracranial aneurysms has evolved from stent-assisted coiling to treatment with densely woven flow-diverting stents. These stents are constructed to redirect the flow though the parent artery and establish a low-flow hemodynamic state within the aneurysm, enabling progressive aneurysm thrombosis and vessel wall remodeling. Similar flow diversion has been accomplished with "regular" intracranial stents by using the stent-in-stent technique. ${ }^{1}$ Two dedicated flow-diverting stents have been developed, the Silk stent (Balt Extrusion, Montmorency, France) and the Pipeline Embolization Device (ev3, Irvine, California). The long-term results for these stents are promising ${ }^{2}$; however, early aneurysm rupture has been described. ${ }^{3}$ The exact cause of the rupture is unknown but might be related to intra-aneurysmal flow and pressure changes. ${ }^{4} \mathrm{We}$, hereby, report the results of pressure measurements inside the aneurysmal sac during placement of a flow-diverting stent by using a dual-sensor pressure and velocity wire. ${ }^{5}$

Received January 7, 2011; accepted after revision February 12.

From the Departments of Radiology (J.J.S, C.B.M, S.P.F, R.v.d.B.) and Biomechanical Engineering and Physics (E.V.), Academic Medical Center, University of Amsterdam, Amsterdam, the Netherlands.

Please address correspondence to J.J. Schneiders, MD, Department of Radiology, G1, Academic Medical Center, University of Amsterdam, PO Box 22660, 1100 DD Amsterdam, the Netherlands; e-mail: j.j.schneiders@amc.uva.nl

http://dx.doi.org/10.3174/ajnr.A2613

\section{TECHNIQUE}

\section{Patient}

A 50-year-old man with a partially thrombosed giant aneurysm $(34 \times 26 \mathrm{~mm})$ of the proximal M1 was referred for endovascular treatment. After careful angiographic analysis, the decision was made to place a flow-diverting stent (Silk, Balt Extrusion) from the ICA to the MCA in conjunction with partial coiling of the aneurysm to promote intra-aneurysmal thrombosis. A slight narrowing in the MCA segment adjacent to the aneurysm was noticed. Clopidogrel (300-mg loading dose, subsequently $75 \mathrm{mg}$ ) and aspirin (400-mg loading dose, subsequently $100 \mathrm{mg}$ ) were started 2 days before the procedure. Thromboaggregation was tested and was adequately blocked.

\section{Description of Technique}

Intra-aneurysmal pressure measurements were performed with a dual-sensor guidewire (ComboWire; Volcano Corporation, Rancho Cordova, California). ${ }^{5}$ The ComboWire features a Doppler velocity sensor and a pressure sensor at the tip of the wire, enabling acquisition of hemodynamic information in a 5-mm-long cone-shaped envelope. The research protocol on measurement of intra-aneurysmal hemodynamics by using the ComboWire was approved by the local ethics committee. The patient met the inclusion criteria, and written informed consent was obtained.

Because it is not possible to navigate a microcatheter through the mesh of a flow-diverting stent, the microcatheter was introduced inside the aneurysm (jailed) before placement of the stent with the intention of placing coils in the aneurysm sac after stent deployment. The jailed microcatheter was used to introduce the ComboWire into the aneurysmal lumen, and continuous intra- 


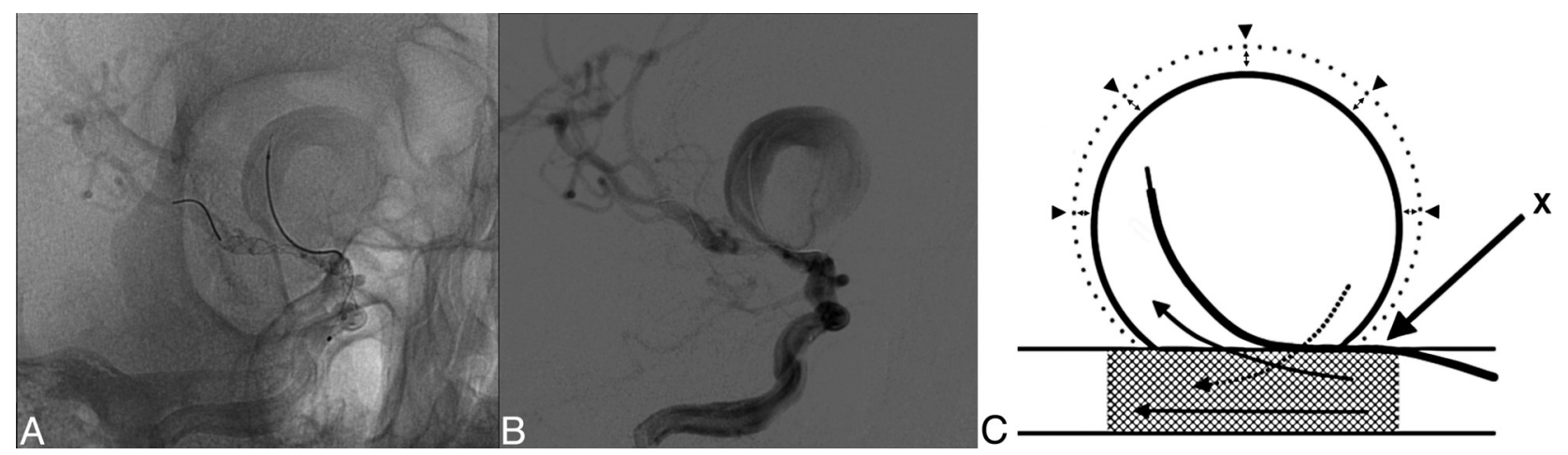

FIG 1. Conventional angiogram $(A)$ and digital subtraction angiogram $(B)$ show the Silk stent deployed in the MCA, and the jailed microcatheter with the ComboWire within the aneurysm. C, Schematic representation of the considered flows (arrows) and aneurysm compliance (arrowheads). $\mathrm{X}$ represents the jailed microcatheter containing the dual-sensor guidewire.

aneurysmal pressure measurements were performed before, during, and after stent deployment (Fig 1).

The measurements were recorded at $200 \mathrm{~Hz}$, resulting in 192,000 data points. Per-point averages of all recorded pressure data were calculated for 30-second periods. These per-point averages were used to determine the mean and maximal pressures. In addition, the pulse pressure (the difference between diastolic and systolic pressure values) was calculated. Flow-velocity measurements were discarded because of noise and unreliability due to complex flow inside the aneurysm.

\section{Procedural Details}

With the patient under general anesthesia, $7 \mathrm{~F}$ and $5 \mathrm{~F}$ sheaths were placed in the right and left femoral arteries, respectively. A 5F guiding catheter was placed in the right ICA. A Tracker Excel-14 microcatheter (Boston Scientific, Fremont, California) was introduced by using a Traxcess guidewire (Microvention, Aliso Viejo, California) for navigation. After selective catheterization of the aneurysm, the Traxcess guidewire was exchanged for the ComboWire. A second guiding catheter was placed in the right ICA to introduce a Vasco +21 MP microcatheter (Balt Extrusion) in the proximal M2 segment of the MCA. The ComboWire was in place inside the aneurysm, and continuous pressure measurements were performed during deployment of the Silk stent $(3.0 \times 25$ $\mathrm{mm})$. Control angiography performed immediately after stent placement showed good patency of both the ICA and MCA (Fig $1)$.

\section{RESULTS}

\section{Mean Pressure}

The mean intra-aneurysmal pressure of $70 \mathrm{~mm} \mathrm{Hg}$ before stent deployment was similar to the systemic mean pressure measured in the femoral artery. During stent deployment, the mean pressure in the aneurysm dropped to $60 \mathrm{~mm} \mathrm{Hg}$, but after complete deployment of the Silk stent, pressure returned to predeployment values and higher (Fig $2 A$ ).

\section{Peak Systolic Pressure}

The peak systolic pressure in the aneurysm before stent deployment was $80 \mathrm{~mm} \mathrm{Hg}$, approximately $20 \mathrm{~mm} \mathrm{Hg}$ lower than the systemic peak systolic pressure measured in the femoral artery. During stent deployment, the peak systolic intra-aneurysmal pressure dropped with $14 \mathrm{~mm} \mathrm{Hg}$ to $66 \mathrm{~mm} \mathrm{Hg}$ with a subsequent steady increase to predeployment values in the next 6 minutes. Systemic peak systolic blood pressure re mained stable between 99 and $105 \mathrm{~mm} \mathrm{Hg}$ during the measurements. Six minutes after the procedure, the peak systolic intra-aneurysmal pressure returned to the baseline values (Fig $2 B$ ).

\section{Pulse Pressure}

During the start of the stent deployment, the pulse pressure decreased sharply from 25 to $13 \mathrm{~mm} \mathrm{Hg}$ but gradually increased during deployment. After complete deployment, the pulse pressure gradually increased to baseline values (Fig $2 \mathrm{C}$ ).

\section{DISCUSSION}

The flow-diverting stent has become a promising device in the treatment of giant and fusiform aneurysms with good short-term outcomes. However, more recent studies also report hemorrhagic complications, due to delayed aneurysm rupture. ${ }^{2,4,6}$ At present, the precise hemodynamic mechanisms leading to aneurysm occlusion through flow diversion are still unclear.

In this study, we measured the intra-aneurysmal pressure before, during, and after placement of a flow-diverting Silk stent. Within minutes after complete stent deployment, intra-aneurysmal pressure returned to baseline values. Peak systolic pressure and pulse pressure were unaffected by the flow diversion, and pressure-related stresses on the aneurysm wall remained similar to those in the untreated situation.

The pressure profile in the aneurysm after deployment of the stent can be described as follows: Pressure in the aneurysm $\left(P_{a n}\right)$ is coupled to its volume $\left(V_{a n}\right)$. Aneurysm compliance $(C)$ is a property of the wall and is defined by the slope of the pressure-volume curve, $C=d V_{a n} / d P_{a n}$.

Simultaneously, net inflow or outflow $(Q)$ through the stent openings depends on the pressure gradient between vessel $\left(P_{v}\right)$ and aneurysm: $Q=\left(P_{v}-P_{a n}\right) / R$, in which $R$ is the resistance of the stent cells. Flow equals the rate of change in volume per definition. Together, stent resistance and aneurysm compliance define a time constant $\tau=R C$. For $\tau$ much larger than the cardiac interval, aneurysm pressure remains constant and equal to the mean local arterial pressure during the cycle. Volume also remains constant, and the wall would be 
protected against cyclic mechanical stress. For a $\tau$ much smaller than the cardiac interval, the aneurysm pressure dynamics are equal to the arterial pressure pulse, and the stent will not affect the cyclic wall stress. With the present flow diverters, it is unclear which range of $\tau$ can be expected.

The current case considers a giant aneurysm, which is expected to have a large compliance, therefore $\tau$ would be high. However, our measurements indicated that the pulse pressure in the aneurysm was equal to the arterial pulse pressure. It seems that flow diverters are too permeable to protect against pulsatile wall stress. While the primary purpose of these stents to reduce inflow and outflow rather than pressure is well-appreciated, they are thus not "pressure diverters."

A possible limitation of our measurements might be the presence of the jailed pressure wire, which may have reduced access resistance through separation of the stent and the vessel wall (Fig 1C). However, this effect is expected to be minimal.

The primary concept of flow diversion as a treatment for intracranial aneurysms is based on stagnation of intra-aneurysmal flow. Immediately after placement of the flow-diverting stent, stagnation of contrast was seen on control angiography, pointing to a reduction on inflow and outflow through the stent cells. This flow reduction does not lead to an immediate intra-aneurysmal thrombosis; consequently, the aneurysm is not protected from pressure-induced stresses. The persisting intraaneurysmal pressure could well be in relation to the already reported (early) rupture of aneurysms after placement of a flow-diverting stent. ${ }^{3,4}$

The concept of flow diversion does not automatically implicate pressure reduction inside the aneurysm. This concept was also proposed in a recent study by Cebral et $\mathrm{al}^{4}$ on computational fluid dynamics simulations of intracranial aneurysms before and after flow diversion. In this study, 3 of 7 patients showed an intraaneurysmal pressure increase, and in 4 of 7 patients, the pressure returned to baseline values after stent placement. None of the patients studied by Cebral et al showed a decrease in intra-aneurysmal pressure after stent placement, which is consistent with our measurements.

We cannot provide data on the course of the intra-aneurysmal pressure in the subsequent time period, but it seems reasonable to suggest that as long as flow is not completely blocked inside the aneurysm, the pulse pressure inside the aneurysm will remain. In the few cases reported, rupture occurred after days to weeks, in the period when one can expect the aneurysm to be still (partially) patent. Kulcsár et $\mathrm{al}^{6}$ proposed that under specific conditions, instead of reverse remodeling and cicatrization of the aneurysm, an aggressive thrombus-associated autolysis of the aneurysm wall can occur, resulting in delayed rupture. The persistence of the pulse pressure inside the aneurysm as determined in this study, in conjunction with the concept of aneurysmal wall autolysis, warrants a very reserved approach and at least second thoughts on the treatment of ruptured aneurysms with flow-diverting stents alone. This is probably also true in those aneurysms with recent growth, in which the aneurysmal wall is probably less stable. This study provides an additional argument that the use of flow diverters for treatment of intracranial aneurysms should be combined with insertion of coils in the aneurysmal sac. Such a recommendation was also made by the manufacturer of the Silk stent in their "Urgent Field Safety Notice." 


\section{CONCLUSIONS}

Although we only investigated a single case, the pressure measurements after placement of a flow diverter may indicate no early reduction of intra-aneurysmal pressure. This means that the aneurysmal wall still has to endure hemodynamic stresses caused by the cardiac pulse wave, which might contribute to rupture in a later phase.

\section{REFERENCES}

1. Benndorf G, Herbon U, Sollmann WP, et al. Treatment of a ruptured dissecting vertebral artery aneurysm with double stent placement: case report. AJNR Am J Neuroradio 2001;22:1844-48

2. Lylyk P, Miranda C, Ceratto R, et al. Curative endovascular reconstruction of cerebral aneurysms with the Pipeline embolization device: the Buenos Aires experience. Neurosurgery 2009;64:632-42, discussion 642-43, quiz N6

3. Turowski B, Macht S, Kulcsár Z, et al. Early fatal hemorrhage after endovascular cerebral aneurysm treatment with a flow diverter (SILK-Stent): do we need to rethink our concepts? Neuroradiology 2011;53:37-41. Epub 2010 Mar 26

4. Cebral JR, Mut F, Raschi M, et al. Aneurysm rupture following treatment with flow-diverting stents: computational hemodynamics analysis of treatment. AJNR Am J Neuroradiol 2011;32: 27-33

5. Ferns SP, Schneiders JJ, Siebes M, et al. Intracranial blood-flow velocity and pressure measurements using an intra-arterial dual-sensor guidewire. AJNR Am J Neuroradiol 2009;31:324-26

6. Kulcsár Z, Houdart E, Bonafé A, et al. Intra-aneurysmal thrombosis as a possible cause of delayed aneurysm rupture after flow-diversion treatment. AJNR Am J Neuroradiol 201 1;32:20-25

7. Largen E. Urgent Field Safety Notice: Intracranial Stent "SILK" Clarification of the Indications. March 2010. http://www.mhra. gov.uk/home/idcplg?IdcService $=$ GET_FILE \&dDocName $=$ CON076110\&RevisionSelectionMethod=LatestReleased(Balt 9-310.pdf) 